\title{
Current Care of Hepatitis C-Positive Patients by Primary Care Physicians in an Integrated Delivery System
}

\author{
David E. Nicklin, MD, Christopher Schultz, MD, Colleen M. Brensinger, MS, and \\ James $P$. Wilson
}

Background: Infection with the hepatitis C virus (HCV) is an emerging health problem in the United States. Management of this condition in asymptomatic patients remains controversial.

Metbods: A questionnaire was mailed in November 1997 to all primary care physicians caring for adults (internists and family physicians) in an integrated health delivery system regarding the current approach to screening, diagnosis, and management of $\mathrm{HCV}$ infection. Charts of patients whose tests were positive for HCV were audited in selected practice sites to document care received by those patients.

Results: Most physicians (70\%) reported ordering alanine aminotransferase (ALT) measurements to screen for HCV infection as part of a complete checkup. Each physician diagnosed an average of 3.1 new cases of HCV infection per year. Patients received widely divergent advice regarding prognosis, precautions to prevent transmission, and treatment. More than one half of the physicians advised their patients that the condition was serious (68\%) and to abstain from alcohol $(56 \%)$ and use condoms in monogamous relationships (62\%). In caring for HCV-positive patients, more than three quarters of physicians reported recommending a liver biopsy to patients who had elevated ALT levels, and observing clinically, without liver biopsy, those patients who had normal ALT levels. A chart audit, however, showed lessaggressive intervention. Approximately one third of HCV-positive patients with elevated ALT levels had been seen by a gastroenterologist and had had a liver biopsy. Physicians in practice longer were less likely to recommend treatment with interferon- $\alpha$. Of those patients whose physicians reported they would recommend biopsy and treatment with interferon- $\alpha$, only $36 \%$ had a documented liver biopsy in their charts, and $29 \%$ had documented interferon- $\alpha$ treatment. Only $1.6 \%$ and $3.0 \%$ of patients, respectively, had received the recommended hepatitis $A$ and hepatitis $B$ vaccines.

Conclusions: Approaches to screening, diagnosis, and management of HCV infection by primary care physicians vary greatly. There appears to be a considerable population of patients in primary care settings who continue to receive conservative management of asymptomatic HCV infection.( $J$ Am Board Fam Pract 1999;12:427-35.)

Hepatitis C virus (HCV) infection is an emerging major health problem in the United States. An estimated 3.9 million persons are chronically infected with $\mathrm{HCV}$, and an estimated 150,000 to 170,000 incident cases are reported annually in the United States. ${ }^{1} \mathrm{HCV}$ infection results in a high proportion $(85 \%)$ of chronic infection. ${ }^{2}$ In patients

Submitted, revised, 3 June 1999

From the Department of Family Practice and Community Medicine (DEN, JPW), the Department of Gastroenterology (CS), and the Center for Clinical Epidemiology and Biostatistics (CB), University of Pennsylvania, Philadelphia. Address reprint requests to David E. Nicklin, MD, Department of Family Practice and Community Medicine, University of Pennsylvania, Mutch Building 6th Floor, 39th and Market St, Philadelphia, PA 19104. referred to academic medical centers, chronic infection results in progressive liver fibrosis leading to cirrhosis in approximately $25 \%$ of patients older than 20 years. $^{2}$ A substantial number of those patients go on to develop liver failure or hepatocellular carcinoma. ${ }^{3}$ The standard treatment for $\mathrm{HCV}$ infection, interferon- $\alpha$, results in long-term clearance of the virus in only a minority of patients. ${ }^{4} \mathrm{~A}$ recent large multicenter, randomized, controlled trial of interferon- $\alpha$ treatment in 912 untreated $\mathrm{HCV}$-positive patients showed a rate of sustained virologic response (defined as HCV RNA undetectable in serum 24 weeks after treatment was completed) of between $6 \%$ and $13 \% .^{5}$ In this trial combining interferon- $\alpha$ with ribavirin increased 
sustained response rates to $31 \%$ (24 weeks of treatment) or $38 \%$ ( 48 weeks of treatment), confirming results of earlier, smaller trials. ${ }^{4,6}$ Even so, $21 \%$ of the patients assigned to combination therapy for 48 weeks discontinued treatment because of severe side effects, a major obstacle to the widespread use of this regimen.

Thus the disease is widespread, chronic, progressive, and without effective treatment in most patients; and it can have serious health consequences. An estimated 8,000 to $10,000 \mathrm{HCV}$-infected patients die of liver disease annually in the United States, ${ }^{7,8}$ which has led some physicians to take an aggressive approach to diagnosis and treatment. A recent cost-benefit analysis and editorial in a major US internal medicine journal suggested that all patients with $\mathrm{HCV}$ infection should undergo liver biopsy, and all those with inflammatory changes on histologic examination should undergo interferon- $\alpha$ therapy. ${ }^{9}$

Others have observed the apparently benign course of the illness in most infected patients. Some estimates of the risk of cirrhosis are as low as $2 \% .^{10}$ A recent report of outcomes in a cohort of 704 women infected 17 years earlier from a common source showed $314(45 \%)$ had cleared the virus and had no detectable HCV RNA in their serum. ${ }^{11}$ Evaluation of 376 patients with persistent infection showed $176(47 \%)$ to have elevated serum alanine aminotransferase (ALT) measurements, but only 7 (2\%) to have definite or probable cirrhosis on liver biopsy, and none had symptoms of liver failure. Of the estimated 3.9 million patients with chronic $\mathrm{HCV}$ infection in the United States, if 10,000 die each year, mortality risk is $0.25 \%$ annually. This finding has led some physicians to take a conservative approach to diagnosis and treatment, reserving liver biopsy and interferon- $\alpha$ therapy for symptomatic patients or those who have evidence of rapid progression of disease. ${ }^{12}$

Counseling patients regarding transmission of $\mathrm{HCV}$, particularly to sexual partners, is also difficult because how the infection is spread is not fully understood. Whereas intravenous drug use, blood transfusion, and occupational exposure to bloodborne pathogens are major identifiable risk factors for $\mathrm{HCV}$ infection in the United States, ${ }^{13}$ the risk of sexual transmission remains uncertain. Less than one half of patients with acute hepatitis $\mathrm{C}$ report a risk factor of parenteral exposure, ${ }^{14}$ suggesting that sexual transmission might play an important role.
Case-control and cross-sectional studies of sexual transmission have yielded conflicting results, with evidence of infection in $0 \%$ to $30 \%$ of patients' sexual partners or spouses. ${ }^{15}$

For example, a cross-sectional study of 50 heterosexual partners (41 women, 9 men) of $\mathrm{HCV}$ positive patients, who had a median duration of sexual relationship of 13 years, showed no transmission. ${ }^{16}$ In a similar study of $100 \mathrm{HCV}$-positive patients and their sexual partners, $17 \%$ of partners were found to be positive for $\mathrm{HCV}$ antibody, and $11 \%$ were infected with the same genotype as their partner. ${ }^{17}$ Transmission was associated with longer duration of the relationship, increased frequency of sexual contact, and shared toothbrushes. A longitudinal study of $449 \mathrm{HCV}$-positive patients and their HCV-negative heterosexual partners for a period of 1 year showed a risk of transmission of 12.0/1000 person-years of exposure. ${ }^{18}$ Transmission was more frequent in older patients who had longstanding sexual relationships. Based on these results, some physicians feel the risk of sexual transmission is negligible, whereas others believe there is a small but undeniable risk.

Although there have been conflicting recommendations in the medical literature regarding diagnosis and management of $\mathrm{HCV}$ infection, in March 1997 the National Institutes of Health released a consensus statement on HCV disease. ${ }^{19}$ This statement suggests that liver biopsy be considered for asymptomatic patients who have elevated serum transaminase levels. We were interested to know whether primary care physicians had adopted this relatively aggressive approach or continued with more conservative management. We therefore undertook a survey of adult primary care physicians in an integrated health delivery system in the United States. We also audited the charts of patients who had $\mathrm{HCV}$ infection diagnosed and who received most of their care at primary care offices. In this way we could document the severity of illness as well as the actual frequency of referral for liver biopsy and interferon- $\alpha$ therapy (as opposed to frequency stated by physicians on the survey).

\section{Methods \\ Dverview}

The study was conducted within an integrated medical delivery system in greater metropolitan 
Philadelphia that provided care annually to approximately 500,000 patients. The system includes approximately 600 academically based, multispecialty physicians and 255 community-based primary care physicians in 108 practice sites. Patients come from a wide range of age and racial groups: $58 \%$ of inpatients are white, $36 \%$ African-American, $0.8 \%$ Hispanic, $0.5 \%$ Asian, and 5\% other; $59 \%$ are female, $41 \%$ male. All physicians practicing general adult medicine or family practice were mailed a letter from the medical director asking their assistance with a written questionnaire. Those not responding were sent a second letter and questionnaire 2 months later.

\section{Sample}

A total of 222 physicians were surveyed, of whom $188(85 \%)$ responded to either the first or second survey request. Physicians seeing patients less than 1 day per week (16 physicians) were excluded, leaving 172 responses for analysis. In addition, we selected all patients who had tested positive for $\mathrm{HCV}$ infection in the past 5 years at the central laboratory facility for the health system, for a total of 1756 patients. We traced the primary care site for these patients through the physician ordering the test as well as the health system billing records for clinical services. Audits were performed on charts of HCVpositive patients whose primary site of care within the health system was a general medicine or family practice office. For logistic reasons, only sites with 5 or more positive patients were included in the chart audit. A total of 186 charts were audited.

\section{Data}

The survey questionnaire elicited information about training, years in practice, and demographics of patients. We asked physicians to report frequency of various interventions in screening, diagnosis, and treatment of HCV infection on a 5-point Likert scale from $0 \%$ (never) to $100 \%$ (always). Chart audits collected information on length of illness, severity of symptoms, and documentation of source of infection, counseling, frequency of referral to specialty care, liver biopsy, and interferon- $\alpha$ treatment.

\section{Analysis}

Data were analyzed using the Statistical Analysis System program..$^{20}$ Survey data were coded, and means with standard deviations calculated for con- tinuous variables and for Likert scale items. Associations were tested by chi-square testing between selected physician characteristics (specialty, years in practice, academic vs community base, urban vs suburban, and racial, insurance, and socioeconomic characteristics of practice) and approach to screening and treatment (eg, ordering screening $\mathrm{ALT}$ measurements, telling patients their life might be shortened, recommending interferon- $\alpha$ therapy, and referral to specialty care). A Mantel-Haenszel chi-square test for trends was used to compare ordinal variables, and a comparison of means for continuous variables was performed using $t$ tests or analysis of variance. The Fisher exact test was used for two-by-two tables, when cells had fewer than five expected counts. Survey responses of physicians recommending specialty consultation, liver biopsy, and interferon- $\alpha$ treatment were compared with actual frequencies of those events in their patients.

\section{Results}

Physician and practice characteristics of respondents are presented in Table 1 and reflect the broad spectrum of the larger metropolitan area. Screening practices among physicians varied widely ( $\mathrm{Ta}$ ble 2). Despite no health screening advisory group recommending routine liver function screening, physicians reported frequently ordering screening ALT measurements, with 120 of $172(70 \%)$ ordering the test on $75 \%$ or $100 \%$ of complete checkups. If patients' ALT levels were elevated, 107 (64\%) of the physicians generally obtained a HCV antibody screening test; 60 (36\%) did not. For patients who reported risk factors for parenteral infection, 36 (21\%) of the physicians responded they routinely tested for the HCV antibody; 132 (79\%) did not. Frequency of new diagnoses of $\mathrm{HCV}$ infection also varied widely, with 48 (28\%) having diagnosed no cases in the past year and $49(29 \%)$ having diagnosed 3 or more cases. (Number responding varies because some physicians did not report a routine practice.)

Physicians also reported a wide variation in their management of HCV infection (Table 2). For example, most physicians reported they told their patients chronic $\mathrm{HCV}$ infection is a serious condition $(80 \%)$ and could result in cirrhosis $(92 \%)$, but only $30 \%$ told their patients it might shorten their life considerably. After making the diagnosis of $\mathrm{HCV}$ infection, $62 \%$ of physicians advised patients 
Table 1. Physician and Practice Characteristics.

\begin{tabular}{lc}
\hline Characteristics & No. (\%) \\
\hline Years in practice & $12.7 \pm 8.9$ \\
Board certification & $160(94)$ \\
Specialty & \\
$\quad$ Family practice & $56(33)$ \\
$\quad$ Internal medicine & $116(67)$ \\
Type of practice & \\
Academic medical center & $30(17)$ \\
Community based & $142(83)$ \\
Location & \\
Urban & $50(33)$ \\
Suburban & $104(67)$ \\
Patients in health maintenance organizations & \\
$<40 \%$ & $46(32)$ \\
$40-59 \%$ & $54(38)$ \\
$\geq 60 \%$ & $42(30)$ \\
African-American patients & \\
$\leq 10 \%$ & $75(50)$ \\
$11-45 \%$ & $49(33)$ \\
$>45 \%$ & $25(17)$ \\
Patients below poverty line & \\
$<10 \%$ & $53(37)$ \\
$10-24 \%$ & $67(47)$ \\
$\geq 25 \%$ & $23(16)$ \\
Hepatitis C cases diagnosed in past year & $3.1 \pm 3.9$ \\
\hline
\end{tabular}

Note-172 physicians responded to the survey; totals in some categories are less than 172 as a result of physician nonresponse to particular questions.

in monogamous relationships to use condoms, $38 \%$ generally did not. Abstinence from alcohol was advised by $56 \%$, while limited alcohol consumption was acceptable to $44 \%$ of physicians. Forty-nine percent of physicians reported routinely referring $\mathrm{HCV}$-positive patients to specialists, whereas $51 \%$ of physicians referred some patients and cared for others without consultation. For HCV-positive patients with normal ALT levels, $30 \%$ of physicians reported recommending liver biopsy, and $21 \%$ recommended treatment with interferon- $\alpha$. When patients' ALT levels were elevated, $82 \%$ recommended a biopsy and $77 \%$ recommended interferon- $\alpha$ treatment. When asked about changes in their management approach, physicians reported they are becoming more aggressive (Table 3). Compared with 12 months ago, $39 \%$ reported they are now more likely to recommend specialty consultation, and $41 \%$ are now more likely to recommend interferon- $\alpha$ therapy, whereas $38 \%$ are now less likely to recommend watchful waiting.
Tests for associations between approaches in screening and physician or practice characteristics were performed, either $t$ test or F test. Physicians with more years in practice were more likely to obtain screening ALT measurements as part of a complete checkup $(P<.001)$. (Table 4) Screening ALT measurements were ordered by $83 \%$ of physicians in practice longer than 15 years. Of those in practice 5 years or less, $43 \%$ also reported routinely performing screening ALT testing.

Although urban address, lower socioeconomic status, and minority ethnic heritage are all associated with increased risk of $\mathrm{HCV}$ infection in studies of blood donors, ${ }^{21}$ our results showed these patients were less likely to be screened. Physicians in community-based practice were more likely to order screening ALT measurements than were those in an academic medical center $(80 \%$ vs $23 \%, P<.001)$. Suburban setting, more white patients, and fewer poor patients remained independently associated with ordering ALT screening measurements after controlling for years in practice (each $P<.01$ ). Suburban setting, more white patients, and fewer poor patients were highly correlated with one another; therefore, the association of each with ordering ALT screening measurements could not be assessed independently on multiple regression analysis.

A stratified analysis showed years in practice, academic vs community practice, and any of the grouped variables of practice location-patient income-patient race were independently associated with ordering screening ALT measurements $(P<.05$ for each, when controlling for other variables). Physicians making the diagnosis frequently (more than 3 cases per year) were no more likely to report ALT screening measurements than those who rarely made the diagnosis ( 0 cases per year) (71\% vs $62 \%, P=.13$ ).

Analysis was performed to determine whether physician or practice characteristics were associated with a more aggressive approach to $\mathrm{HCV}$ infection management. Frequency of recommending treatment for asymptomatic HCV-positive patients who had normal serum transaminase results was low overall $(20 \%)$ and not associated with years in practice $(P=.91)$, inner-city vs suburban practice $(P=.54)$, percentage of low-income patients $(P=.59)$, or numbers of new HCV infection diagnoses per year $(P=86)$. Physicians recommended treatment more frequently for $\mathrm{HCV}$-positive pa- 
Table 2. Physicians' Reported Approach to Screening, Diagnosis, and Management of Hepatitis C Virus (HCV) Infection.

\begin{tabular}{|c|c|c|c|c|c|c|}
\hline \multirow[b]{2}{*}{ Interventions } & \multicolumn{5}{|c|}{ Estimate of Percentage of Patients so Advised } & \multirow[b]{2}{*}{$\underset{\text { SD }}{\operatorname{Mean}} \pm$} \\
\hline & $\begin{array}{c}0 \% \\
(=0) \\
\text { No. }(\%)\end{array}$ & $\begin{array}{c}25 \% \\
(=1) \\
\text { No. }(\%)\end{array}$ & $\begin{array}{c}50 \% \\
(=2) \\
\text { No. }(\%)\end{array}$ & $\begin{array}{c}75 \% \\
(=3) \\
\text { No: }(\%)\end{array}$ & $\begin{array}{c}100 \% \\
(=4) \\
\text { No. }(\%)\end{array}$ & \\
\hline Screening $A L T$ as part of complete examination & $21(12.2)$ & $14(8.1)$ & $17(9.9)$ & $36(20.9)$ & $84(48.8)$ & $2.9 \pm 1.4$ \\
\hline $\begin{array}{l}\text { Obtain HCV antibody testing on patients with } \\
\text { elevated ALT levels }\end{array}$ & $9(5.4)$ & $20(12.0)$ & $31(18.6)$ & $59(35.3)$ & $48(28.7)$ & $2.7 \pm 1.2$ \\
\hline $\begin{array}{l}\text { Obtain HCV antibody testing on patients with } \\
\text { risk factors }\end{array}$ & $40(23.8)$ & $36(21.4)$ & $34(20.2)$ & $22(13.1)$ & $36(21.4)$ & $1.9 \pm 1.5$ \\
\hline \multicolumn{7}{|l|}{$\begin{array}{l}\text { Tell HCV-positive patients (physician with no } \\
\text { diagnosis of } \mathrm{HCV} \text { in past year excluded) }\end{array}$} \\
\hline HCV infection is a serious medical condition & $11(9.1)$ & $13(10.7)$ & $15(12.4)$ & $19(15.7)$ & $63(52.1)$ & $2.9 \pm 1.4$ \\
\hline HCV infection can markedly shorten life & $39(32.8)$ & $24(20.2)$ & $18(15.1)$ & $10(8.4)$ & $28(23.5)$ & $1.7 \pm 1.6$ \\
\hline HCV infection generally progresses very slowly & $11(9.5)$ & $13(11.2)$ & $10(8.6)$ & $28(24.1)$ & $54(46.6)$ & $2.9 \pm 1.4$ \\
\hline Irreversible liver damage could be present & $26(21.8)$ & $23(19.3)$ & $20(16.8)$ & $18(15.1)$ & $32(26.9)$ & $2.1 \pm 1.5$ \\
\hline HCV infection can result in cirrhosis & $2(1.7)$ & $8(6.6)$ & $12(9.9)$ & $18(14.9)$ & $81(66.9)$ & $3.4 \pm 1.0$ \\
\hline Risk of cirrhosis is great & $22(18.5)$ & $19(16.0)$ & $19(16.0)$ & $20(16.8)$ & $39(32.8)$ & $2.3 \pm 1.5$ \\
\hline $\mathrm{HCV}$ infection can result in liver cancer & $24(20.3)$ & $23(19.5)$ & $11(9.3)$ & $15(12.7)$ & $45(38.1)$ & $2.3 \pm 1.6$ \\
\hline Risk of liver cancer is serious & $53(44.5)$ & $22(18.5)$ & $20(16.8)$ & $8(6.7)$ & $16(13.4)$ & $1.3 \pm 1.4$ \\
\hline Recommend interferon- $\alpha$ therapy & $12(10.1)$ & $16(13.4)$ & $29(24.4)$ & $20(16.8)$ & $42(35.3)$ & $2.5 \pm 1.4$ \\
\hline Do not share razors or toothbrushes & $20(17.1)$ & $8(6.8)$ & $9(7.7)$ & $7(6.0)$ & $73(62.4)$ & $2.9 \pm 1.6$ \\
\hline Use condoms if monogamous & $35(29.9)$ & $5(4.3)$ & $4(3.4)$ & $6(5.1)$ & $67(57.3)$ & $2.6 \pm 1.8$ \\
\hline Use condoms if multiple partners & $9(7.5)$ & $3(2.5)$ & $1(0.8)$ & $5(4.2)$ & $102(85.0)$ & $3.6 \pm 1.2$ \\
\hline Minimize alcohol consumption & $8(6.7)$ & $4(3.3)$ & $5(4.2)$ & $8(6.7)$ & $95(79.2)$ & $3.5 \pm 1.2$ \\
\hline Abstain from alcohol & $32(27.1)$ & $12(10.2)$ & $8(6.8)$ & $8(6.8)$ & $58(49.2)$ & $2.4 \pm 1.8$ \\
\hline Have partners checked for $\mathrm{HCV}$ infection & $7(5.8)$ & $9(7.5)$ & $8(6.7)$ & $12(10)$ & $84(70.0)$ & $3.3 \pm 1.2$ \\
\hline Receive hepatitis A vaccine & $66(55.5)$ & $9(7.6)$ & $10(8.4)$ & $4(3.4)$ & $30(25.2)$ & $1.4 \pm 1.7$ \\
\hline Receive hepatitis B vaccine & $34(28.6)$ & $12(10.1)$ & $11(9.2)$ & $4(3.4)$ & $58(48.7)$ & $2.3 \pm 1.8$ \\
\hline
\end{tabular}

Mean score range: never $=0$, always $=4$; a difference in means of 1.0 reflects $25 \%$ more patients given that advice.

ALT-alanine transaminase

tients who had elevated serum transaminases (77\%). Physicians with fewer years in practice were more likely to recommend treatment $(P=.01)$. There was no association between recommending treatment and inner-city vs suburban practice $(P=.30)$, percentage of low-income patients $(P$
$=.38$ ), or number of new cases of HCV infection diagnosed $(P=.45)$. There were also no significant differences between family physicians and internists in recommending treatment of $\mathrm{HCV}$ infection $(P=.57)$ or between those with fewer or more managed health care $(\mathrm{HMO})$ patients $(P=.59)$.

Table 3. Referral Recommendations and Change in Practice in the Past Year.

\begin{tabular}{lr}
\hline Characteristics & No. (\%) \\
\hline Referral recommendations & $90(81.8)$ \\
If HCV positive and ALT levels are elevated, recommend liver biopsy & $80(76.9)$ \\
If HCV positive and ALT levels elevated, recommend interferon- $\alpha$ therapy & $74.9 \% \pm 32 \%$ \\
Estimate percentage of HCV-positive patients referred to gastroenterologist & $49(31.2)$ \\
Change in practice in past year-more likely to & $63(40.6)$ \\
Recommend liver biopsy & $63(39.1)$ \\
Recommend interferon- $\alpha$ therapy & \\
Refer to subspecialist &
\end{tabular}

$\mathrm{HCV}$-hepatitis C virus, $\mathrm{ALT}$-alanine transaminase. 
Table 4. Frequency of Ordering Routine Alanine Aminotransferase Measurements.

\begin{tabular}{|c|c|c|c|}
\hline Practice Characteristics & $\begin{array}{c}\text { Mean } \\
\text { Likert } \\
\text { Scale Value }\end{array}$ & $P$ Value $^{\star}$ & $\begin{array}{c}\text { Controlling for } \\
\text { Years in Practice } \\
P \text { Value* }\end{array}$ \\
\hline \multicolumn{4}{|l|}{ Type of practice } \\
\hline Academic medical center & $1.4 \pm 1.5$ & $<.001$ & $<.001$ \\
\hline Community based & $3.2 \pm 1.2$ & & \\
\hline \multicolumn{4}{|l|}{ Location } \\
\hline Urban & $2.0 \pm 1.6$ & $<.001$ & .01 \\
\hline Suburban & $3.2 \pm 1.1$ & & \\
\hline \multicolumn{4}{|l|}{ African-American patients } \\
\hline$\leq 10 \%$ & $3.2 \pm 1.2$ & $<.001$ & .001 \\
\hline $11-45 \%$ & $2.8 \pm 1.4$ & & \\
\hline$>45 \%$ & $1.6 \pm 1.6 \mathfrak{x}$ & & \\
\hline \multicolumn{4}{|l|}{ Patients below poverty line } \\
\hline$<10 \%$ & $3.2 \pm 1.2$ & .016 & .02 \\
\hline $10-24 \%$ & $2.8 \pm 1.5$ & & \\
\hline$\geq 25 \%$ & $2.2 \pm 1.6$ & & \\
\hline
\end{tabular}

${ }^{\star} P$ values by $t$ test or $F$ test.

Note: mean score range: never $=0$, always $=4$; difference in means of 1.0 reflects $25 \%$ more patients screened.

Chart audits of $\mathrm{HCV}$-positive patients at primary care sites with 5 or more $\mathrm{HCV}$-positive patients yielded 186 patients, $79 \%$ of whom had elevated ALT levels. Of the $186,102(55 \%)$ had been seen by a gastroenterologist, $51(27 \%)$ had documentation of liver biopsy, and $37(20 \%)$ had received interferon- $\alpha$ therapy. Three patients (2\%) had documentation of receiving hepatitis A vaccine, and $6(3 \%)$ had received hepatitis $B$ vaccine. Because there were significantly fewer patients being cared for by specialists and receiving liver biopsy than expected from survey responses, a further analysis was restricted to patients of physicians who had responded to the survey. Eighty-three patients were cared for by physicians who had responded to the survey (48 physicians). Physicians had indicated on the questionnaire whether they recommend liver biopsy or interferon- $\alpha$ therapy for their $\mathrm{HCV}$ positive patients who had normal ALT levels and for those who had elevated ALT levels. Each patient was then classified according to whether a biopsy and interferon- $\alpha$ treatment had been recommended, depending upon his or her ALT result and physician's stated practice in responding to the survey. Results are shown in Table 5 and Table 6.

\section{Discussion}

A great percentage of physicians (70\%) reported routinely ordering screening ALT measurements as part of a complete physical examination. This finding was somewhat surprising, as such testing is not recommended by any health screening guidelines. Specifically, the Report of the US Preventive Services Task Force makes no mention of routine laboratory screening for liver dysfunction in their comprehensive report and guidelines. ${ }^{22}$ Physicians

Table 5. Physician Reports Biopsy Recommended vs Chart Shows Biopsy Performed.

\begin{tabular}{lcc}
\hline & \multicolumn{2}{c}{$\begin{array}{c}\text { Patient Chart Shows Liver } \\
\text { Biopsy Performed on } \\
\text { Patient }\end{array}$} \\
\cline { 2 - 3 } $\begin{array}{l}\text { Recommend Liver Biopsy } \\
\text { in Survey Response }\end{array}$ & $\begin{array}{c}\text { Yes } \\
\text { No. (\%) }\end{array}$ & No \\
\hline Yes & $21(36)$ & $38(64)$ \\
No & $5(21)$ & $19(79)$ \\
\hline
\end{tabular}

Table 6. Physician Reports Interferon- $\alpha$ Therapy Recommended vs Chart Shows Interferon- $\alpha$ Therapy Received.

\begin{tabular}{|c|c|c|}
\hline \multirow[b]{2}{*}{$\begin{array}{l}\text { Recommend Interferon- } \alpha \\
\text { Therapy in Survey Response }\end{array}$} & \multicolumn{2}{|c|}{$\begin{array}{l}\text { Patient Chart Shows } \\
\text { Interferon- } \alpha \text { Therapy } \\
\text { Received by Patient }\end{array}$} \\
\hline & $\begin{array}{c}\text { Yes } \\
\text { No. (\%) }\end{array}$ & $\begin{array}{c}\text { No } \\
\text { No. }(\%)\end{array}$ \\
\hline Yes & $17(29)$ & $41(71)$ \\
\hline No & $2(8)$ & $23(92)$ \\
\hline
\end{tabular}


in practice longer were more likely to order ALT measurements, as were those in suburban settings, with fewer poor and minority patients. Patients known to be at higher risk for $\mathrm{HCV}$ infection (minority, poor, and inner-city) were less likely to be screened. Possible explanations include financial incentives, education or training, and patient expectations. Physicians in the system are neither financially penalized nor rewarded for laboratory utilization, so financial incentives do not appear to be important. That physicians longer in practice were more likely to order ALT measurements might be due to practice changes that occurred since their training; however, almost one half of recently trained physicians frequently order this test. A third explanation would be a tendency for physicians to order additional tests to assure a complete examination, with the experience that patients are pleased and reassured by normal results. This phenomenon has been documented in routine testing done preoperatively and at hospital admission (electrocardiogram, chest radiograph, prothrombin and partial thromboplastin times). ${ }^{23-25}$ Physicians in suburban, more affluent settings who order screening ALT measurements more frequently might be responding to a more demanding patient population.

With regard to follow up screening when ALT levels were elevated, $36 \%$ of physicians reported not testing for $\mathrm{HCV}$ antibody in most of these patients. This finding was unexpected because chronic HCV infection would be a serious possibility for asymptomatic patients with elevated ALT levels. Possible explanations for physicians' failing to pursue the diagnosis of $\mathrm{HCV}$ infection might include its uncertain natural history, lack of effective treatment for the disease, and a belief that alcohol use or fatty infiltration of liver are more likely causes. Only $35 \%$ of physicians reported generally testing those patients for $\mathrm{HCV}$ infection who had a history of high-risk behavior, possibly for similar reasons. Despite the uncertainty of prognosis and the lack of effective treatment for most patients at this time, however, the NIH consensus panel on $\mathrm{HCV}$ infection recommends testing asymptomatic at-risk patients. ${ }^{18}$ It appears that for now most primary care physicians have not adopted that approach.

Physicians reported wide variations in approach to counseling HCV-positive patients. Many of these differences reflect uncertainty regarding transmission, prognosis, and management. In some areas where there is clear agreement, however, practice recommendations could be improved. Sharing razors poses a risk of transmission, but $32 \%$ of physicians do not advise against it. Recent reports suggest both hepatitis A and hepatitis B vaccines should be offered routinely. Fewer than one half of physicians routinely recommend these vaccinations, and chart audit shows them being received by less than $5 \%$ of patients. Abstinence from alcohol has been reported to slow progression of hepatic fibrosis, but only $56 \%$ of physicians recommend it. Interestingly, within this system, although physicians longer in practice were more likely to use ALT measurements to screen for $\mathrm{HCV}$ infection, they were less likely to recommend interferon- $\alpha$ treatment to $\mathrm{HCV}$-positive patients. Perhaps the more recent graduates have more experience with interferon- $\alpha$ treatment. Alternatively, more experienced physicians might be more reluctant to prescribe a treatment that has considerable toxicity and a low chance of cure for patients who are feeling well and have a good prognosis.

If ALT levels were elevated in HCV-positive patients, most physicians (82\%) reported recommending liver biopsy. If ALT levels were normal, most physicians $(70 \%)$ reported recommending no biopsy and continued monitoring. This management strategy is consistent with the spirit of the guidelines set forth by the NIH consensus panel on $\mathrm{HCV}$ infection, which recommends a less aggressive approach when caring for patients who have persistently normal ALT levels. ${ }^{19}$ Overall, physicians estimated they referred $75 \%$ of their HCVpositive patients for consultation. Chart audit, however, showed only a minority of patients had documented biopsy or treatment. Explanations for this low level of adherence include physicians actually referring patients less often than stated, patients disregarding physician recommendations, and consultation, biopsy, or treatment being received by patients but not documented in chart.

Chart audit was restricted to patients receiving most of their care in primary care offices of the system; patients who were cared for primarily in the gastroenterology or in the transplant clinic were excluded. As a result, the charts audited were those of patients who had less severe disease not requiring aggressive management, which might, in part, explain the low percentage of patients receiving consultation, liver biopsy, and interferon- $\alpha$ treat- 
ment. Patients who are feeling well would be less likely to undergo an invasive procedure that has a small but serious risk so they can qualify for a treatment that is not likely to result in long-term clearance of the virus.

It appears there is a large group of patients with $\mathrm{HCV}$ infection (roughly one half of those being cared for in primary care offices) who are doing well and have not been examined by a gastroenterologist. Whether a more complete evaluation confers an increased benefit to patients and whether the substantial group of patients who have not received biopsies should be aggressively pursued and referred will be clearer as the medical establishment gains a greater understanding of the natural history of the disease and as more effective treatment is developed. The absence of these patients (as well as those who are asymptomatic and whose disease is undiagnosed) from studies of the natural history of the disease reported from specialty centers might have resulted in a selection bias in which patients with rapidly progressive disease are largely overrepresented.

\section{Summary}

A survey of primary care physicians in an integrated delivery system showed considerable variation in screening, diagnosis, and treatment of $\mathrm{HCV}$ disease. Most physicians routinely screened for $\mathrm{HCV}$ infection by measuring patients' ALT levels, which is not recommended by guidelines. Physicians in practice longer and those in affluent, suburban areas were more likely to screen for $\mathrm{HCV}$ infection. A minority of physicians followed up patients with abnormal ALT results or those at risk for parenterally transmitted disease with recommended $\mathrm{HCV}$ antibody testing. Widely divergent advice was given to patients regarding prognosis, need for condom use in monogamous relationships, and other precautions to prevent transmission, reflecting the uncertainty in the medical literature in those areas.

When caring for $\mathrm{HCV}$-positive patients, more than three quarters of physicians reported recommending liver biopsy to patients with elevated ALT levels, observing without liver biopsy those with normal ALT levels. Physicians longer in practice were less likely to recommend treatment. Chart audit showed that only one third of patients for whom biopsy was reportedly recommended re- ceived liver biopsy, and most stable HCV-positive patients had not received comprehensive evaluation or treatment. Areas of potential improvement in care highlighted by the survey include reducing use of screening ALT tests, increasing use of $\mathrm{HCV}$ antibody testing in at-risk patients, routinely counseling $\mathrm{HCV}$-positive patients to abstain from alcohol, and recommending hepatitis $A$ and $B$ vaccines.

\section{References}

1. Alter MJ. Epidemiology of hepatitis C. Hepatology 1997;26(3 Suppl 1):62S-65S.

2. Poynard T, Bedossa P, Opolon P. Natural history of liver fibrosis progression in patients with chronic hepatitis C. The OBSVIRC, METAVIR, CLINIVIR, and DOSVIRC groups. Lancet 1997;349:82532.

3. Tsukuma $H$, Hiyama $T$, Tanaka S, et al. Risk factors for hepatocellular carcinoma among patients with chronic liver disease. N Engl J Med 1993;328:1797801.

4. Lai MY, Kao JH, Yang PM, et al. Long-term efficacy of ribavirin plus interferon alpha in the treatment of chronic hepatitis C. Gastroenterology 1996;111: 1307-12.

5. McHutchison JG, Gordon SC, Schiff ER, et al. Interferon alfa-2b alone or in combination with ribavirin as initial treatment for chronic hepatitis C. Hepatitis Interventional Therapy Group. N Engl J Med 1998;339:1485-92.

6. Reichard O, Norkrans G, Fryden A, Braconier JH, Sonnerborg A, Weiland O. Randomized, doubleblind, placebo-controlled trial of interferon alpha-2b with and without ribavirin for chronic hepatitis $C$. The Swedish Study Group. Lancet 1998;351:83-7.

7. Alter MJ, Mast EE, Moyer LA, Margolis HS. Hepatitis C. Infect Dis Clin North Am 1998;12:13-26.

8. Recommendations for prevention and control of hepatitis $\mathrm{C}$ virus (HCV) infection and $\mathrm{HCV}$-related chronic disease. Centers for Disease Control and Prevention. MMWR Morb Mortal Wkly Rep 1998; 47(RR-19): 1-39.

9. Bennett WG, Inoue $Y$, Beck JR, Wong JB, Pauker SG, Davis GL. Estimates of the cost-effectiveness of a single course of interferon-alpha $2 \mathrm{~b}$ in patients with histologically mild chronic hepatitis $C$. Ann Intern Med 1997;127:855-65.

10. Management of hepatitis C. Hepatology 1997;26(3 Suppl 1):S1-S155.

11. Kenny-Walsh E. Clinical outcomes after hepatitis $C$ infection from contaminated anti-D immune globulin. Irish Hepatology Research Group. N Engl J Med 1999;340:1228-33.

12. Levine R. Treating histologically mild chronic hepatitis $\mathrm{C}$ : monotherapy, combination therapy, or tincture of time? Ann Intern Med 1998;129:323-6. 
13. Alter MJ, Margolis HS, Krawczynski K, et al. The natural history of community-acquired hepatitis $\mathrm{C}$ in the United States. The Sentinel Counties Chronic non-A, non-B Hepatitis Study Team. N Engl J Med 1992;327:1899-905.

14. Alter MJ. The detection, transmission, and outcome of hepatitis $C$ virus infection. Infect Agents Dis 1993;2:155-66.

15. Alter MJ. Transmission of hepatitis $C$ virus-route, dose, and titer. N Engl J Med 1994;330:784-6.

16. Bresters D, Mauser-Bunschoten EP, Reesink HW, et al. Sexual transmission of hepatitis $C$ virus. Lancet 1993;342:210-1.

17. Kao J, Hwang YT, Chen PJ, et al. Transmission of hepatitis $\mathrm{C}$ virus between spouses: the important role of exposure duration. Am J Gastroenterol 1996;91: 2087-90.

18. Piazza M, Sagliocca L, Tosone G, et al. Sexual transmission of the hepatitis $C$ virus and efficacy of prophylaxis with intramuscular immune serum globulin. A randomized controlled trial. Arch Intern Med 1997;157:1537-44.

19. National Institutes of Health Consensus Development Conference Panel statement: management of hepatitis C. In: Management of hepatitis C. Hepatology 1997;26(3 Suppl 1):2S-10S. Also available at http://consensus.nih.gov

20. Statistical analysis system, version 6.12. Cary, NC: SAS Institute, 1998.

21. Bodsworth NJ, Cunningham P, Kaldor J, Donovan B. Hepatitis $C$ virus infection in a large cohort of homosexually active men: independent associations with HIV-1 infection and injecting drug use but not sexual behavior. Genitourin Med 1996;72:118-22.

22. Guide to clinical preventive services: report of the US Preventive Services Task Force. 2nd ed. Baltimore: Williams \& Wilkins, 1996.

23. Wachtel TJ, O'Sullivan P. Practice guidelines to reduce testing in the hospital. J Gen Intern Med 1990;5:335-41.

24. Narr BJ, Hansen TR, Warner MA. Preoperative laboratory screening in healthy Mayo patients: costeffective elimination of tests and unchanged outcomes. Mayo Clin Proc 1991;66:155-9.

25. Marcello PW, Roberts PL. "Routine" preoperative studies. Which studies in which patients. Surg Clin North Am 1996;76:11-23. 